\title{
Buckling Instability in Liquid Crystalline Physical Gels
}

\author{
Rafael Verduzco, ${ }^{1}$ Guangnan Meng, ${ }^{2}$ Julia A. Kornfield, ${ }^{1}$ and Robert B. Meyer ${ }^{2}$ \\ ${ }^{1}$ California Institute of Technology, MC 210-41,Pasadena, California 91125, USA \\ ${ }^{2}$ The Martin Fisher School of Physics, Brandeis University, Waltham, Massachusetts 02454-9110, USA \\ (Received 1 December 2005; revised manuscript received 28 February 2006; published 13 April 2006) \\ In a nematic gel we observe a low-energy buckling deformation arising from soft and semisoft elastic \\ modes. We prepare the self-assembled gel by dissolving a coil-side-group liquid-crystalline polymer- \\ coil copolymer in a nematic liquid crystal. The gel has long network strands and a precisely tailored \\ structure, making it ideal for studying nematic rubber elasticity. Under polarized optical microscopy we \\ observe a striped texture that forms when gels uniformly aligned at $35^{\circ} \mathrm{C}$ are cooled to room temperature. \\ We model the instability using the molecular theory of nematic rubber elasticity, and the theory correctly \\ captures the change in pitch length with sample thickness and polymer concentration. This buckling \\ instability is a clear example of a low-energy deformation that arises in materials where polymer network \\ strains are coupled to the director orientation.
}

DOI: $10.1103 /$ PhysRevLett.96.147802

PACS numbers: $61.30 .-\mathrm{v}, 61.41 .+\mathrm{e}, 82.70 . \mathrm{Gg}$

Liquid-crystalline elastomers and gels combine the orientational order of liquid crystals and the translational order of a polymer network. The coupling of these two effects in liquid-crystalline elastomers and gels gives rise to unique phenomena such as soft elasticity in which certain director rotations and network strains cost no free energy in the ideal case. Soft elastic modes are predicted and well understood theoretically by a molecular theory of nematic rubber elasticity [1-3]. However, a significant limitation in testing existing theories is the difficulty in preparing well-characterized networks. Many approaches have been undertaken to prepare liquid crystal elastomers, including free-radical polymerization of bulk liquid crystal acrylates and diacrylates [4], free-radical polymerization of diacrylates in the presence of liquid crystals $[5,6]$, and side-coupling reactions with poly(methylsiloxane) [7]. Unfortunately, these approaches result in nematic elastomers and gels with heterogeneities in the polymer network and, in the case of many gels, phase separation of the polymer network from the liquid crystal host [8,9]. More recently, self-assembly of block copolymers has been used as an approach to create model liquid crystal networks [10-12]. We have utilized self-assembly of a triblock copolymer that has a narrow molar mass distribution and a very long midblock to produce well-defined nematic gels $[10,11]$. This gel facilitates comparison to present theories which assume the material is lightly cross-linked and single phase.

In this Letter, we present a study of the effects of nematic rubber elasticity in thin layers of uniformly aligned gels contained between rigid parallel plates. Using polarized optical microscopy, we observe a banded texture that arises in response to small temperature changes. We propose that this texture represents a buckling deformation made possible by the presence of soft and semisoft elastic modes. We formulate a description based on the molecular theory of nematic elastomers that incorporates soft and semisoft elasticity as well as liquid crystal elasticity, also known as Frank elasticity. The theory correctly accounts for the observed dependence of the pitch and threshold temperature on the sample thickness and polymer concentration.

The self-assembled gels are prepared by dissolving a high molecular weight $\left(\sim 1200 \frac{\mathrm{kg}}{\mathrm{mol}}\right)$ polystyrene-sidegroup liquid-crystalline polymer-polystyrene triblock copolymer in the nematic liquid crystal 4-cyano-4'-npentylbiphenyl (5CB) (Fig. 1) [10,11]. The polystyrene block is considerably less soluble than the liquidcrystalline block in $5 \mathrm{CB}$, and it aggregates to form physical cross-links. The resulting self-assembled network has a uniform molecular weight between cross-links.

In this work, we chose to study gels with polymer concentrations of $10 \mathrm{wt} \%$ or less where the polymer rigidity is important but not dominant. Samples were loaded into cells with well-defined gap spacings and alignment layers. For gaps smaller than $10 \mu \mathrm{m}$, we used cells purchased from LC Vision. The cells consist of indium-tinoxide coated glass plates with a layer of $\mathrm{SiO}_{2}$ to induce planar alignment. For gaps larger than $10 \mu \mathrm{m}$, we used glass slides with teflon spacers and a rubbed polyimide layer for alignment. The cell thickness was determined

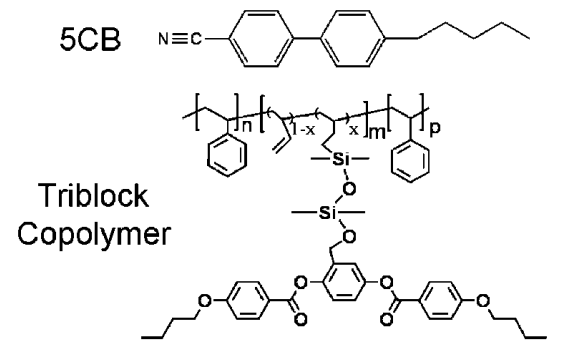

FIG. 1. Chemical structure of liquid crystal 5CB and the endassociating triblock copolymer used in this study. The midblock is a random copolymer of liquid crystal side-group and butadiene monomer. The polydispersity $\left(\frac{M_{w}}{M_{n}}\right)$ of the triblock is approximately 1.6 as measured by gel-permeation chromatography. 
with a uv-visible spectrophotometer by interferometry for gaps smaller than $10 \mu \mathrm{m}$ [13] or by measuring the thickness with a micrometer for larger gaps. The nematic gels have a sol-gel transition temperature that is approximately equal to the nematic-isotropic phase transition temperature $\left(T_{\mathrm{NI}}\right)$ of $5 \mathrm{CB}, 35.2^{\circ} \mathrm{C}$, and the gel loads easily by capillary action when heated above this temperature.

A magnetic field or shear are used to uniformly align the gel. In the first case, the gel is placed in an $8.8 \mathrm{~T}$ magnetic field oriented parallel to the desired director orientation, heated above its $T_{\mathrm{NI}}$, and allowed to cool to room temperature. After removing the cell from the magnetic field, the cell is heated to $34^{\circ} \mathrm{C}$ and then cooled back down to room temperature at $1{ }^{\circ} \mathrm{C} / \mathrm{min}$ to remove any residual irregularities. In some cases it is easier to use shear to align the sample. This is because alignment by magnetic field is more sensitive to bubbles and defects in the sample. In order to shear align the sample, the sample is loaded into a Mettler FP 82 hot stage and the temperature is set to $34.5^{\circ} \mathrm{C}$ while the top slide is carefully moved back and forth across the sample.

Gels that are uniform monodomains at $35^{\circ} \mathrm{C}$ develop a striped texture when cooled to room temperature (Fig. 2). The texture reversibly disappears and reappears when the gel is heated above and then cooled below a threshold temperature for stripe formation. Below this threshold temperature, the pitch length is independent of temperature. The stripes run perpendicular to the initial director orientation $\underline{n}_{o}$. Rotating the crossed polarizers to approximately $\pm 15^{\circ}$ maximizes contrast between dark and bright bands, and the stripes that appear dark at $+15^{\circ}$ appear bright at $-15^{\circ}$ and vice versa. The stripes are less evident but still visible when the polarizers are aligned with $\underline{n}_{o}$. This indicates that the director rotates within the plane and in opposite directions in adjacent stripes but does not preclude an out-of-plane rotation of the director coupled with this in-plane rotation.

The characteristics of the striped texture depend on the sample thickness and polymer concentration. The pitch is larger in thicker samples and more dilute gels (Fig. 3). Stripes do not appear when the gap is too thin or the
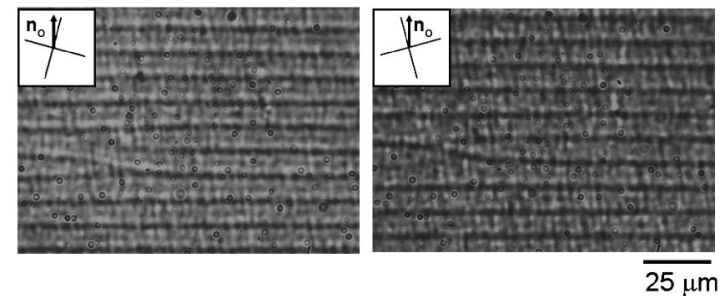

FIG. 2. A 5 wt. $\%$ gel in a $25 \mu \mathrm{m}$ gap at $20.0^{\circ} \mathrm{C}$ viewed under a polarizing optical microscope. The orientation of the director imprinted during cross-linking $\underline{n}_{O}$ and the orientation of the crossed polarizers is shown in the top left-hand corner of each image. Note that the dark lines in the left-hand image become bright in the right-hand image and vice versa. polymer concentration too dilute. In the case of a $5 \mathrm{wt}$ \% gel, stripes do not appear in 4 and $9 \mu \mathrm{m}$ thick cells but they do appear at larger gap thicknesses. For $10 \mathrm{wt} \%$ gels, stripes appear even in thin cells. Stripe formation is thus favored in larger gaps and more concentrated gels.

A periodic modulation of the director is unexpected because it introduces a free energy contribution from Frank elasticity. [A periodic striped texture has been observed in very thin $(<1 \mu \mathrm{m})$ nematic films due to surface gradient terms in the elastic free energy expression [14,15]. Our samples are thick enough that the surface gradient terms are not significant.] The appearance of the stripes can be accounted for, however, by including the rubber elastic energy of the polymer network. A side-chain liquidcrystalline polymer dissolved in a nematic solvent adopts an anisotropic chain conformation coupled to the nematic order of the solvent [16]. Based on prior literature on nematic elastomers [1] we expect that an unconstrained freestanding gel will change shape: in the case of a strongly prolate alignment tendency, the gel will expand in the direction of director orientation with decreased temperature (our gel is too soft to carry out the same experiment). When constrained between glass plates, however, expansion of the gel in the direction of increasing anisotropy is prevented by the boundary conditions, and the network becomes increasingly frustrated as the temperature decreases. The stripe instability represents a low-energy deformation incorporating a periodic network strain field coupled to a periodic rotation of the director. The deformation reduces the rubber elastic energy relative to the undeformed state at the cost of a Frank elastic penalty.

We formulate a molecular model of the instability by treating the gel as a nematic elastomer, as has been previously done successfully $[1,17]$. We start with a macroscopically uniform nematic gel in which the director $\underline{n}$ is aligned in a unique direction parallel to the substrates of a dielectric cell. The polymer strands in the gel have an anisotropic conformation described by a step length tensor $l_{i j}=l_{\perp} \delta_{i j}+\left(l_{\|}-l_{\perp}\right) n_{i} n_{j}$ where $\delta_{i j}$ is the identity tensor. The network anisotropy $r$ is defined as the ratio $\frac{l_{\|}}{l_{\perp}}$. The anisotropy $r$ is a temperature dependent property that in-

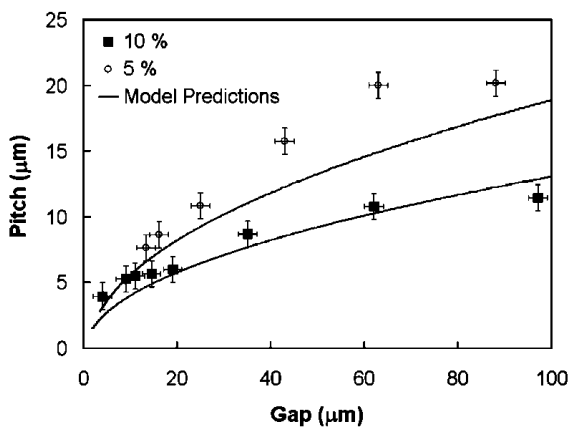

FIG. 3. Dependence of pitch on gap in 10 and 5 wt. \% polymer gels. 
creases with decreasing temperature for our system. The anisotropy during cross-linking is denoted as $r_{o}$.

Below a critical temperature, $r$ becomes sufficiently large that the gel buckles. The buckling instability represents a director rotation coupled to a network strain field. We assume that the director stays in the $x-y$ plane and that the director orientation depends on $x$ and $z$ but is independent of $y$ (Fig. 4). Coupled to this director rotation is a network strain field described by the deformation gradient tensor $\lambda_{i j}$. At the interface with the cell $\left(z= \pm \frac{d}{2}\right)$, the substrates impose the boundary conditions of no-slip and that $\underline{n}$ must be aligned along $x$.

The free energy density consists of three terms: ideal polymer network elasticity, nonideal polymer network elasticity, and Frank elasticity [Eq. (1)]. The ideal nematic elastic term is weighted by $\mu$. The nonideal contribution is weighted by $\mu \alpha$ and describes the semisoft elasticity observed experimentally [3]. Semisoftness arises from compositional fluctuations and from a memory of the aligned state imprinted during cross-linking. The last term is the Frank elastic term. We make the one-constant approximation [18] with Frank elastic constant $K$.

$$
\begin{aligned}
F= & \frac{1}{2} \mu\left(\operatorname{Tr}\left[\underline{\underline{l_{o}}} \cdot \underline{\underline{\lambda}}^{T} \cdot \underline{\underline{l}}^{-1} \cdot \underline{\underline{\lambda}}\right]\right) \\
& +\frac{1}{2} \mu \alpha\left(\operatorname{Tr}\left[\left(\underline{\underline{\delta}}-\underline{n}_{o} \underline{n}_{o}\right) \cdot \underline{\underline{\lambda}}^{T} \cdot \underline{n} \underline{n} \cdot \underline{\lambda}\right]\right) \\
& +\frac{1}{2} K\left[(\nabla \cdot \underline{n})^{2}+(\underline{n} \cdot \nabla \times \underline{n})^{2}+(\underline{n} \times \nabla \times \underline{n})^{2}\right] .
\end{aligned}
$$

We analyze the behavior near the transition to the striped state where we assume small values for the amplitudes of the director rotation and the displacements. We propose simple sinusoidal functions for the director rotation $\phi$ and the displacement field $\underline{\Delta}$ [Eqs. (2)], with $\Delta_{i}$ denoting the displacement along the $i$ axis. These functions vary sinusoidally in the $x$ direction with wave vector $k$, and the wave vector $q$ has the value $\frac{\pi}{d}$ so that the director and displacement fields satisfy both the homogeneous boundary condition and the no-slip boundary condition at the substrates $\left(z= \pm \frac{d}{2}\right)$. The terms $\xi$ and $\gamma$ set the amplitudes of the rotation and displacement fields, respectively.

$$
\begin{aligned}
\phi(x, z) & =\xi \cos (q z) \cos (k x), \\
\Delta_{y} & =\gamma \cos (q z) \sin (k x), \quad \Delta_{x}=\Delta_{z}=0 .
\end{aligned}
$$

We calculate the deformation gradient tensor $\underline{\lambda}$ from the displacement field $\left(\lambda_{i j}=\delta_{i j}+\frac{\partial \Delta_{i}}{\partial x_{j}}\right)$. Substitution of $\underline{\lambda}$ and
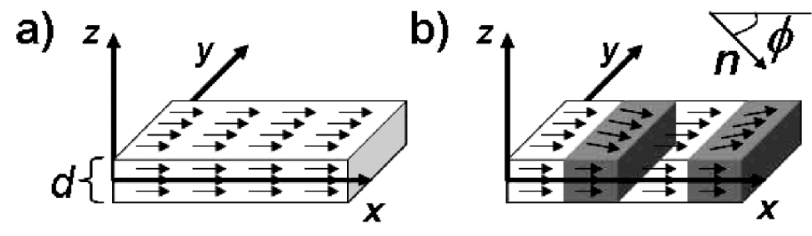

FIG. 4. Nematic gel director orientation in (a) the initial uniformly aligned state and (b) the striped state. The cell substrates are at $z= \pm \frac{d}{2}$. $\underline{n}$ into Eq. (1) gives the free energy density of an incompressible gel subject to the periodic deformation and director rotation described by Eqs. (2). We Taylor expand this function in $\xi$ around $\xi=0$ and keep only terms of $\mathrm{O}\left(\xi^{2}\right)$ or greater. Next, we integrate the simplified free energy density over one wavelength of the deformation to obtain the average free energy density of the gel. Subtracting the energy of the uniformly aligned gel from this free energy gives the free energy relative to the monodomain state [Eq. (3)].

$$
\begin{aligned}
F= & \frac{1}{8} K \xi^{2}\left(k^{2}+q^{2}\right)+\mu\left\{\frac{1}{8} \gamma^{2}\left(q^{2}+k^{2} r_{o}\right)+\frac{r_{o}}{4} \gamma \xi k\left(\frac{1}{r}-1\right)\right. \\
& \left.+\frac{1}{8} \xi^{2}\left(r_{o}-1+\alpha+\frac{1}{r}-\frac{r_{o}}{r}\right)\right\} .
\end{aligned}
$$

A crossing from a positive to a negative value in the free energy indicates an onset of the buckling instability. The first term in Eq. (3) is the contribution from Frank elasticity and is always positive, as expected. The contribution from nonideal network elasticity, the term weighted by $\alpha$, is also always positive and increases the threshold to the striped state. In a semisoft gel (nonzero $\alpha$ ) a small but finite cooling is always required for the appearance of the instability. The soft elastic part contributes negative terms to the free energy expression which are responsible for the appearance of the buckling instability.

In order to compute an anisotropy and wave vector, we look for the minimum value of $r$ and a critical wave vector $k$ for which the right combination of infinitesimal displacement and rotation costs zero energy, signalling an instability as $r$ passes through this value. For slightly larger $r$ values, the amplitude of the instability grows rapidly, with the wavelength determined at the instability.

We do not know the values of many of the material parameters in the model, but they can be reasonably approximated from independent measurements or from known properties of the liquid crystal solvent 5CB. For example, the liquid crystal elastic constants have been measured previously for $5 \mathrm{CB}$ at room temperature $[13,19]$ and have values of about $1 \times 10^{-11} \mathrm{~N}$. We measured the rubber modulus $\mu$ in a cone-and-plate rheometer and obtained a value of approximately $220 \mathrm{~Pa}$ for a 10 wt. $\%$ gel and $50 \mathrm{~Pa}$ for a 5 wt. $\%$ gel $[10,11]$. We set the initial anisotropy $r_{o}$ to the small value of 1.2. In addition to these parameters, we reasonably approximate the semisoftness parameter $\alpha$ to have a value of 0.05 based on previous studies of nematic elastomers [17].

A comparison of the model prediction of the pitch and experimental results shows good qualitative agreement (Fig. 3). The model predicts the trends of increasing pitch with increasing gap and decreasing polymer concentration. The model also accounts for the disappearance of stripes at small gaps from a prediction of the threshold anisotropy (Fig. 5). Neutron scattering measurements show the anisotropy of the present midblock at $25^{\circ} \mathrm{C}$ to be 10 [10]. 


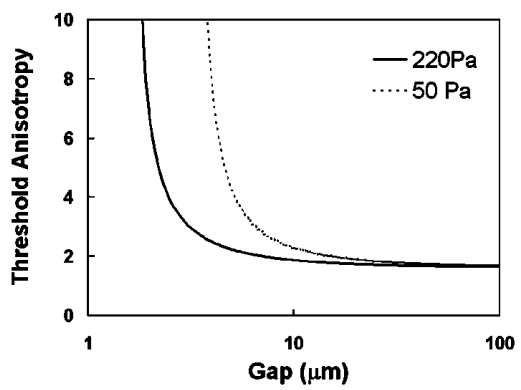

FIG. 5. Predicted value of the threshold anisotropy $r_{\text {th }}$ as a function of gap thickness $d$ and rubber modulus $\mu$. Bands form if the polymer has $r>r_{\text {th }}$. The values of $\mu$ correspond to those of the 5 and $10 \mathrm{wt} \%$ gels.

Therefore, the model predicts that bands are suppressed for $d<3.7 \mu \mathrm{m}$ for the $5 \mathrm{wt}$. \% gel and for $d<1.9 \mu \mathrm{m}$ for the 10 wt. $\%$ gel.

In very thick samples ( $q \approx 0)$, the buckling instability is a soft mode that carries no penalty from nematic rubber elasticity. This can be seen in the free energy expression [Eq. (3)]: in the limit $q \rightarrow 0$, the negative contribution $\sim r_{o} \gamma \xi k\left(\frac{1}{r}-1\right)$ can always compensate the positive contributions for any $k$ by properly selecting $\gamma$ and $\xi$. When the sample has a finite thickness, however, the boundary conditions necessitate gradients from the center to the walls of the gap. The resulting gradient $\frac{\partial \Delta_{y}}{\partial z}-$ a shearing deformation - is not a soft mode. Nevertheless, the deformation is semisoft, and the cost to nematic rubber elasticity is small. The reason that the elastic cost is small is also shown by Eq. (3): by selecting a large spacial frequency $k$, the positive contribution from the $\gamma^{2} q^{2}$ term is negligible and the negative term can compensate for the positive elastic contribution with a sufficiently large amplitude of director rotation $\xi$. Thus, in the absence of Frank elasticity, the gel would favor a small wavelength distortion.

However, Frank elasticity goes inversely with the square of the wavelength, making very short wavelengths costly in energy. The final wavelength is a compromise of nematic rubber elasticity and Frank elasticity. The final wavelength is set by the rubber modulus $\mu$, the Frank elastic constant $K$, the initial anisotropy $r_{o}$, and the semisoftness parameter $\alpha$.

We have presented a stripe distortion in nematic gels and shown that it arises due to the coupling of orientational and translational degrees of freedom in nematic elastomers. A model based on the molecular theory of nematic rubber elasticity accounts for the observed experimental features: the onset of the instability with changes in the network anisotropy, the increase in the pitch with increasing sample thickness, the decrease in the pitch with increasing polymer concentration, and the disappearance of the instability at small sample thicknesses.

This work was supported by the Department of Defense, the James Irvine Foundation, AFOSR LC-MURI (F4962097-1-0014), NSF Grant No. DMI-0523083, the W. M. Keck Foundation, and at Brandeis NSF Grant No. DMR0322530.

[1] M. Warner and E. M. Terentjev, Liquid Crystal Elastomers (Oxford University Press, Oxford, 2003).

[2] M. Warner, P. Bladon, and E. M. Terentjev, J. Phys. II (France) 4, 93 (1994).

[3] H. Finkelmann, I. Kundler, E. M. Terentjev, and M. Warner, J. Phys. II (France) 7, 1059 (1997).

[4] D. J. Broer, H. Finkelmann, and K. Kondo, Makromol. Chem. 189, 185 (1988).

[5] R. A. M. Hikmet, J. Appl. Phys. 68, 4406 (1990).

[6] C.-C. Chang, L.-C. Chien, and R. B. Meyer, Phys. Rev. E 56, 595 (1997).

[7] J. Kupfer and H. Finkelmann, Makromol. Chem., Rapid Commun. 12, 717 (1991).

[8] A. Jakli, D. R. Kim, L. C. Chien, and A. Saupe, J. Appl. Phys. 72, 3161 (1992).

[9] P. Gautier, M. Brunet, J. Grupp, L. Noirez, and E. Anglaret, Phys. Rev. E 68, 011709 (2003).

[10] M. D. Kempe, N. R. Scruggs, R. Verduzco, J. Lal, and J. A. Kornfield, Nat. Mater. 3, 177 (2004).

[11] M.D. Kempe, R. Verduzco, N.R. Scruggs, and J.A. Kornfield, Soft Matter (to be published).

[12] M.-H. Li, P. Keller, J. Yang, and P.-A. Albuoy, Adv. Mater. 16, 1922 (2004).

[13] I.-C. Khoo and S.-T. Wu, Optics and Nonlinear Optics of Liquid Crystals (World Scientific, Singapore, 1993).

[14] O. D. Lavrentovich and V. M. Pergamenshchik, Phys. Rev. Lett. 73, 979 (1994).

[15] V. M. Pergamenshchik, Phys. Rev. E 61, 3936 (2000).

[16] M. D. Kempe, J. A. Kornfield, and J. Lal, Macromolecules 37, 8730 (2004).

[17] E. M. Terentjev, M. Warner, R.B. Meyer, and J. Yamamoto, Phys. Rev. E 60, 1872 (1999).

[18] P. G. DeGennes and J. Prost, The Physics of Liquid Crystals (Clarendon Press, Oxford, 1993).

[19] K. Skarp, S. T. Lagerwall, and B. Stebler, Mol. Cryst. Liq. Cryst. 60, 215 (1980). 